\title{
Urgences
}

\section{Cœur du domaine}

\section{Renald Bérubé et André Gervais}

Numéro 19, janvier 1988

Le tour du texte

URI : https://id.erudit.org/iderudit/025445ar

DOI : https://doi.org/10.7202/025445ar

Aller au sommaire du numéro

Éditeur(s)

Urgences

ISSN

0226-9554 (imprimé)

1927-3924 (numérique)

Découvrir la revue

Citer ce document

Bérubé, R. \& Gervais, A. (1988). D. Cœur du domaine. Urgences, (19), 52-72.

https://doi.org/10.7202/025445ar d'utilisation que vous pouvez consulter en ligne.

https://apropos.erudit.org/fr/usagers/politique-dutilisation/ 


\section{Coeur du domaine:}

prototexte

pré-texte

texte

paléotexte: voir texte

textilité

textualité

textualiste: voir textualité

homotextualité

textualisation, textualiser

métatextualisation

homotextualisation

hétérotextualisation

syntextualisation

cotextualisation

métatextème

antitexte

sociotexte

contexte

contexture

grammatextualité

callitexte

textanalyse

textologie, textologique

textologue: voir textologie

texte source [texte souche, texte matrice]

texte de base: voir texte source

texte cible (texte objet] 


\section{Prototexte}

RÉF. - Walter Geerts, 1980 - «Pour une herméneutique structurale". "Les chats" de Baudelaire. Une confrontation de méthodes, Namur et Paris, Presses universitaires de Namur et PUF, 1980, p. 305-325.

> > Un autre article sur le même poème de Baudelaire (Léon Somville: "Le poème "Les chats" de Baudelaire, essai d'exégèse», publié dans Études littéraires, Québec, vol. 5, no 2, août 1972), repris dans le recueil cité plus haut, indique que "proto-poème", terme à partir duquel sera formé prototexte, est proposé par Charles Mauron dans son livre intitulé Le dernier Baudelaire (Paris, Corti, 1966, p. 85-86). La définition que nous choisissons est donc celle que propose L. Somville de protopoème.

DÉF. - «Par «proto-poème», Mauron entend bien désigner, chez Baudelaire en particulier, cet agrégat d'images surgies de l'inconscient, antérieures à l'écriture mais obstinées à s'y infiltrer selon un processus qui se dérobe à l'attention de l'écrivain, comme à celle de la plupart de ses lecteurs...»

Léon Somville: «Le poème "Les chats" de Baudelaire, essai d'exégèse», dans Maurice Delcroix et Walter Geerts (sous la direction de): «Les chats" de Baudelaire. Une confrontation de méthodes, Namur et Paris, Presses universitaires de Namur et PUF. 1980, p. 227.

>> Michel Grimaud («Psychologie et littérature», dans Aron Kibédi Varga (sous la direction de): Théorie de la littérature. Coll. "Connaissance des langues», Paris, Picard, 1981, p. 268) écrit ceci: "Enfin, s'inspirant d'Anton Ehrenzweig et d'Ernst Kris, Colin Martindale ([«Psychological Contributions to Poetics» (Poetics, 7, 1978, pp. 121-133)]) note que la création n'est pas la mise en place d'un texte, manifeste résultant, par déformation, d'un * sous-texte latent et logique - mais bien plutôt de l'élaboration d'un proto-texte que nous qualifierions volontiers de sensori-moteur et préopératoire.» Sauf erreur, le sous-texte dont il est question ici est plutôt le * pré-texte selon N. Celeyrette-Pietri.

\section{Pré-texte}

RÉF. - Nicole Celeyrette-Pietri, 1977; François Rigolot, 1982

DÉF. - Il s'agit, au départ d'un texte, «du choix des contraintes, des algorithmes et de la stratégie». 
Nicole Celeyrette-Pietri: "Le con(mp)te comp(n)te». Littérature. Paris, no 28, décembre 1977, p. 102.

«Sous le texte, il faut entendre le pré-texte, le modèle abstrait qui a voulu le structurer».

Nicole Celeyrette-Pietri: «Le roman de la statue. Le sujet de l'énonciation d'après les fragments narratifs de Valéry", dans Jean Decotignies (sous la direction de): Les sujets de lécriture, Lille, Presses universitaires de Lille, 1981, p. 237.

>> Faut-il souligner que le terme ainsi défini serait un proche parent de ${ }^{*}$ prototexte et de ${ }^{*}$ génotexte?

$\S$ «Si l'on appelle * hors-texte la référentialité du texte, c'est-à-dire le monde des choses par rapport auxquelles s'écrit le livre, on désignera par pré-texte l'ensemble des conditions de production qui entourent l'oeuure et lui donnent son sens en tel ou tel point de l'aire culturelle. Le débat déjà ancien qui a opposé une «nouvelle» critique à une "ancienne" critique a masqué le fait que les adversaires ne se divisaient pas à propos du *hors-texte mais du pré-texte. L'ancien historien de la littérature s'intéresssait à l'oeuure, à ses conditions de production et de représentation, alors que le nouveau critique soumettait le texte à des pratiques «scientifiques» (sociologie, psychanalyse, etc.) qui devaient, en principe, neutraliser l'ingérence du prétexte. On pourrait résumer l'effort de ce que fut la «nouvelle critique» en disant qu'elle visait à mettre l'oeuure en position de texte tandis que son aînée avait cru pouvoir, jusque-là, se passer du texte pour restituer le sens de l'oeuure dans sa totalité."

François Rigolot: «La Renaissance du texte. Histoire et sémiologie». Poétique. Paris, no 50, avril 1982, p. 191.

>> Faut-il souligner que pré-texte, dans cette autre acception, serait un proche parent de la * textuation?

\section{Texte}

REF. - Julia Kristeva, 1966-1967; Philippe Sollers, 1968; Jean Ricardou, 1968; Roland Barthes, 1970 et 1973; Jean Bellemin-Noël, 1972; François Rigolot, 1982; Bernard Magné, 1986

DÉF. - «Plus qu'un discours, la sémiotique se donne actuellement pour objet plusieurs pratiques sémiotiques qu'elle considère comme translinguistiques, c'est-à-dire faites à travers la langue et irréductibles aux catégories qui lui sont, de nos jours, assignées.

Dans cette perspective, nous définissons le texte comme un appareil translinguistique qui redistribue l'ordre de la langue, en 
mettant en relation une parole communicative visant l'information directe, avec différents types d'énoncés antérieurs ou synchroniques. Le texte est donc une productivité, ce qui veut dire: 1 . son rapport à la langue dans laquelle il se situe est redistributif (destructivoconstructif), par conséquent, il est abordable à travers des catégories logiques plutôt que purement linguistiques; 2 . il est permutation de textes, une ${ }^{*}$ intertextualité: dans l'espace d'un texte plusieurs énoncés, pris à d'autres textes, se croisent et se neutralisent.»

Julia Kristeva: Sèméiôtikè. Recherches pour une sémanalyse. Coll. «Tel Quel».Paris, Seuil, 1969, p. 113.

+ «[... Parl texte, j'entends ici non seulement l'objet saisissable par l'impression de ce qu'on appelle un livre (un roman), mais la totalité concrète à la fois comme produit déchiffrable et comme travail d'élaboration transformateur. En ce sens, la lecture et l'écriture du texte font à chaque reprise partie intégrante du texte qui, d'ailleurs, se calcule en conséquence. Il s'agit donc d'un texte ouvert donnant sur un texte généralisé $(. .$.$) .$

|...

Le texte comporte ainsi trois niveáux principaux:

1. une couche profonde: «l'écriture» comme mise en scène et englobement de la représentation (traces, marques, nombres) (nombres $=$ "battement artériel des choses" - Artaud);

2. une couche intermédiaire: l'* intertextualité, ce que nous avons appelé le corps matériel (qui relance la fonction narrative);

3. une couche superficielle: mots, rimes, phrases, séquences, "motifs» etc. (écriture) (un "motif» est un ensemble de mots actifs) le tout formant une sorte d'accumulateur dynamique qui se génère de 1 à 3 et se déchiffre de 3 à 1 .

On peut dire, par conséquent, que dans ce type de texte tout est "écrit": la constitution du texte, sa structuration, ses niveaux de sens, ses ellipses, ses suspensions, ses intervalles, ses jonctions, sa trame.» Philippe Sollers: «Niveaux sémantiques d un texte moderne», dans Tel Quel (sous la direction de): Théorie densemble. Coll, «Tel Quel», Paris, Seuil, 1968, p. 318 et 324.

+ «Le texte n'est pas un espace neutre où viennent s'assembler des sens inaltérables, c'est un milieu de transformation, une machine à changer les sens.»

Jean Ricardou: "Fonction critique", dans Tel Quel (sous la direction de): Théoric densemble. Coll. «Tel Quel», Paris, Seuil, 1968, p. 261.

+ «ll y a d'un côté ce qu'il est possible d'écrire et de l'autre ce qu'il n'est plus possible d'écrire: ce qui est dans la pratique de l'écrivain et ce qui en est sorti: quels textes accepterais-je d'écrire (de ré-écrire), de désirer, davancer comme une force dans ce monde qui est le mien? Ce que l'évaluation trouve, c'est cette valeur-ci: ce qui peut être aujourd'hui écrit (ré-écrit): le scriptible. Pourquoi le scriptible est-il 
notre valeur? Parce que l'enjeu du travail littéraire (de la littérature comme travail), c'est de faire du lecteur, non plus un consommateur, mais un producteur du texte. Notre littérature est marquée par le divorce impitoyable que l'institution littéraire maintient entre le fabricant et l'usager du texte, son propriétaire et son client, son auteur et son lecteur. (...)

Le texte scriptible est un présent perpétuel, sur lequel ne peut se poser aucune parole conséquente (qui le transformerait, fatalement, en passé); le texte scriptible, c'est nous en train d'écrire, avant que le jeu infini du monde (le monde comme jeu) ne soit traversé, coupé, arrêté, plastifié par quelque système singulier (Idéologie, Genre, Critique) qui en rabatte sur la pluralité des entrées, l'ouverture des réseaux, l'infini des langages. (...) Interpréter un texte, ce n'est pas lui donner un sens (plus ou moins fondé, plus ou moins libre), c'est au contraire apprécier de quel pluriel il est fait.»

Roland Barthes: S/Z. Coll. «Tel Quel», Paris, Seuil, 1970, p. 10-11.

>> Le texte scriptible s'oppose au texte lisible, à «ce qui peut être lu, mais non écrit: le lisible. Nous appelons classique tout texte lisible." (p. 10). Jean Ricardou (Esprit, Paris, no 12 (no intitulé Lecture l: l'espace du texte), décembre 1974, p. 786) appelle paléotexte tel type de texte lisible: "Or, si la littérature ancienne se laisse penser (je ne dis pas correctement) dans l'idéologie de l'expression, la littérature moderne rend caduque toute cette conception. À cet égard, le texte de Soljénitsyne s'affiche comme l'expression d'un quelque chose à dire; ce qui du point de vue où je me place ici, lui confère une importance très secondaire, parce qu'il propose de l'ancien dans la période moderne, parce qu'il est ce que j’appelle du paléo-texte.».

«Le texte ne doit pas être confondu avec l'oeuvre. Une oeuvre est un objet fini, computable, qui peut occuper un espace physique (prendre place par exemple sur les rayons d'une bibliothèque); le texte est un champ méthodologique; on ne peut donc dénombrer (du moins régulièrement) des textes; tout ce qu'on peut dire, c'est que, dans telle ou telle oeuvre, il y a (ou il n'y a pas) du texte: «L'oeuvre se tient dans la main, le texte dans le langage.» On peut dire d'une autre façon que, si l'oeuvre peut être définie en termes hétérogènes au langage (allant du format du livre aux déterminations sociohistoriques qui ont produit ce livre), le texte, lui, reste de part en part homogène au langage: il n'est que langage et ne peut exister qu'à travers un autre langage. Autrement dit, "le texte ne s'éprouve que dans un travail, une production": par la signifiance. (...)

La signifiance, qui est le texte au travail, ne reconnait pas les domaines imposés par les sciences du langage (ces domaines peuvent être reconnus au niveau du ${ }^{*}$ phéno-texte, mais non à celui du * géno-texte); la signifiance - lueur, fulguration imprévisible des 
infinis du langage — est indistinctement à tous les niveaux de l'oeuvre |... . . [...

Bien plus: on ne peut, en droit, restreindre le concept de «texte» à l'écrit (à la littérature). Sans doute la présence de la langue articulée (ou, si l'on préfère: maternelle) dans une production donne à cette production une richesse plus grande de signifiance; très construits, puisque issus d'un système très codé, les signes langagiers s'offrent à une déconstruction d'autant plus percútante; mais il suffit qu'il y ait débordement signifiant pour qu'il y ait texte: la signifiance dépend de la matière (de la "substance") du signifiant seulement dans son mode d'analyse, non dans son être. [...] Toutes les pratiques signifiantes peuvent engendrer du texte: la pratique picturale, la pratique musicale, la pratique filmique, etc.»

Roland Barthes: "Texte (théorie du)» Itexte écrit en 19731. Encyclopaedia Universalis. Paris, 1980. p. 1015-1016.

+ «1) par rapport à l’* avant-texte, c'est le texte "définitif» ou plus exactement le dernier état d'une élaboration, signé par l'écrivain. Il fonctionne comme le terme - mais non le but - d'une suite de transformations matérielles, terme décidé (tranché) par le mouvement de l'écriture. En fait, n'étant encore lui-même qu'une étape aléatoire, il ne saurait récuser l"* avant-texte comme «non-texte».

2) par rapport à loeuure ou au poème, c'est l'écrit en tant que matérialisation, support et foyer d'une écriture et d'une lecture, considéré en dehors de toute référence au «sujet» producteur, au «sujet» consommateur et aux conditions de publication (un recueil, un genre, voire l'ensemble de la Littérature). Par définition le texte sera ici un écrit qui se replie sur lui-même, lieu de rencontre de deux opérations, écriture et lecture, qui le constituent conjointement. C'est un ensemble de signes qui ne "renvoient» qu'à eux-mêmes et à leur organisation hic et nunc. Contrairement au poème qui est un repaire $\mathrm{de}^{*}$ contextes, le texte n'a pas de ${ }^{*}$ contexte - à l'exception peut-être de son * avant-texte, dans la mesure où celui-ci se trouve suffisamment hors de lui pour être avec lụi. Le texte n'est pas un objet, posé là une fois pour toutes, pourvu d'une finalité précise, habité par un sens qui l'aurait déterminé totalement: il serait plutôt un moment, instable, et un lieu, provisoire; ou encore un point stratégique dont le prix tient aux conditions de son occupation et dont les coordonnées varient selon la conjoncture. Le penser - en fait: le traverser, le manipuler, car il n'est guère "pensable» - exige qu'on passe d'une doctrine rationaliste de type mécaniste-positiviste à une attitude phénoménologique et dialectique, selon laquelle il fonctionne comme «pratique», se constituant et se transformant sans cesse, n'ayant dautre origine ni d'autre existence que le procès même de sa constitutiontransformation."

Jean Bellemin-Noël:Le texte etlavant-texte. Coll. «L», Paris, Larousse, 1972, p. 17-18. 
+ «Ainsi la variation générique sur textus et textum permet d'introduire une duplicité sémantique du texte, à la fois composé et non composé, signe achevé et forme ouverte, monument et document.

En un sens, le texte de la Renaissance occupe (ou cherche à occuper) la béance qui s'ouvre entre ce masculin (textus) et ce neutre [textum]. Le parcours qui va de la callida junctura de la poétique horacienne à la compositio incomposita de la rhétorique quintilienne se trouve repris et rejalonné par l'histoire du texte. Entre Chrétien de Troyes et Montaigne, entre le «roman» et l' «essai», la "bele conjointure» est devenue une «ingénieuse contexture». Certes la philologie rend compte à retardement de l'évolution sémantique d'un concept qui, lui aussi, opère sa translatio à travers des textes. La formulation réflexive des poétiques et des rhétoriques est d'ailleurs toujours décalée par rapport à la praxis littéraire.»

"François Rigolot: «La Renaissance du Texte. Histoire et sémiologie». Poétique. no 50. avril 1982. P. 192-193.

>> > Le sens, ici, de contexture est celui qu'il a au XVle siècle, tel que le rappelle F. Rigolot dans cet article (p. 188): «Pour l'auteur des Essais, la "contexture» peut désigner soit la disposition de l'homme soit celle du discours. [...] À la «bele conjointure» de Chrétien de Troyes correspond la "belle contexture» de Montaigne; seulement le sens est inversé: l'emploi mélioratif est devenu ironique dans les Essais $(\ldots) . »$

+ «Le texte sera ici considéré comme un écrit (c'est-à-dire un énoncé graphique résultat d'une scription) soumis à un surcodage par rapport au fonctionnement de la langue (la mise en oeuvre de ce surcodage étant appelé écriture).»

Bernard Magné: «Métatextuel et lisibilité». Prolée. Chicoutimi, vol. 14, no I-2 (no intitulé La lisibilité), printemps-été 1986, p. 77.

>> > Jean Bellemin-Noël (Littérature, Paris, no 28, décembre 1977, p. 13) définit ainsi scription: "Scription" n'est ni graphie (modelé à peu près permanent lié à une main écrivante) ni rédaction (mouvement d'une réalisation lié à un projet d'écriture) ni, bien sùr, écriture (unité offerte à la lecture d'une forme et d'un sens): plutôt l'ensemble des conditions de rédaction qui sont aux donnés textuels inscrits sur le papier ce que l'énonciation est à l'énoncé, soit la manifestation du sujet écrivant. Ou plus exactement: les manifestations, car il s'en repère mainte espèce.» 


\section{Textilité}

RÉF. - Jean Ricardou, 1978

DÉF. - « «... il s agira d' un texte, c'est-à-dire d une texture, d'un tissu. Non point, donc, d'une ligne, mais d'un entrecroisement de lignes. Cependant, nous le savons, tout cela, une fois de plus, aujourd'hui, est une métaphore. Textilement, le tissu est en quelque façon une surface intrinsèque: celle-ci tient par elle-mème en ce quelle est issue, si l'ont peut dire, de deux dimensions, la trame et la chaîne. Typographiquement, le texte est en quelque façon une surface extrinsèque: celle-ci ne tient que par le parasitage d'une page en ce qu'elle est faite d'une dimension unique, la ligne d'écriture, découpée en segments égaux inscrits parallèlement. Cette superficielle ressemblance n'est ainsi guère en mesure de permettre, par elle-même, la métaphore qui associe au tissu ce qu'on nomme communément le texte: elle forme seulement la condition d'une similitude plus accomplie. En effet, ce qui caractérise le texte, c'est que chacun de ses éléments tend à s'y voir pourvu d'un double système de relations: d'une part, celui qui rive l'élément à sa place, selon ce que nous appelons, suivant l'aspect à souligner, les relations littérales ou linéaires; d'autre part, celui qui lie cet élément à certains passages éloignés, selon ce que nous appelons les relations translittérales ou translinéaires. C'est la dénomination métaphorique de ces deux types de liaison selon le lexique de la surface qui fait intervenir les relations longitudinales ou horizontales (linéaires) et transversales ou verticales (translinéaires), dont l'entrecroisement, dès lors, simule bien une certaine textilité."

Jean Ricardou: Nouveaux problèmes du roman. Coll. «Poétique». Paris. Seuil, 1978. p. 245.

\section{Textualité}

RÉF. - Jean Ricardou, 1978

DÉF. - «Nommons textualité d'un élément son aptitude à figurer dans tel état (de * textilité) d'un texte (...). En conséquence, bien que ceci puisse paraître renversant à certains; il faut le reconnaitre: avec ce principe, ce qui choisit les éléments de tel état du texte, ce n'est plus l'aveuglant quelque chose à dire; c'est plutôt, ( ....) en l'état où il est, le texte même. Ce choix des éléments, en ce qu'il s'accomplit à partir des multiples liaisons permises par le texte, nous proposons de lappeler surdétermination textuelle. Ce nombre des liaisons qui 
astreignent tel élément à tel état du texte, nous proposons de le nommer coefficient de surdétermination.. ».

Jean Ricardou: Nouveaux problèmes du roman. Coll. «Poétique», Paris. Seuil, 1978. p. 245-246.

>> > Raymonde Debray-Genette ("Génétique et théories littéraires", dans Louis Hay et Peter Nagy (sous la direction de): Avant-texte, texte, après-texte. Colloque international de textologie [Matrafüred, octobre 1978I, Paris, CNRS et Budapest, Akadémiai Kiado, 1982, p. 167-168) propose dappeler textualiste celui qui est préoccupé par l'analyse de la textualité du texte: «Les rapports actuels qu'entretiennent et doivent entretenir les études génétiques et la critique littéraire me semblent permettre de préciser deux notions liées entre elles, mais qui conduisent à des pratiques différentes, la notion de textuel, celle qui préoccupe les * textologues, et la notion de textualité ou encore de littérarité, qui préoccupe les textualistes. (...) Si la critique intègre la génétique, et l'inverse, elles se rendront des services réciproques. Les textologues apprendront aux textualistes la relativité de la critique et les plaisirs de la science, et les textualistes aux textologues la relativité de la science et les plaisirs de la critique.»

\section{Homotextualité}

\section{RÉF. - Jean-Pierre Dubost, 1986}

DÉF. — "ll semble donc quau moment où (...) la technologie tend à se libérer rigoureusement de toute généralisation du mécanique, de toute extériorité extra-machinique, le texte de Jarry [Le surmâle], tout en répétant le même mouvement de stricte limitation - puisque toutes les figures essentielles du machinique jarryque sont des figures de l'autosuffisance - ce texte fait tout pour tenir en l'air comme le globe dans sa giration, le désir homosexuel et androgyne entrant en parfaite homologie avec une homolextualité. opérant ainsi une illimitation inverse puisque la machine textuelle (c'est-à-dire la cohésion rigoureuse de paquets symboliques maintenus dans un bain de phrases d'une extrême viscosité et faisant par là signes entre eux, entrant ensemble en giration), aboutit à accoupler perversement le machinal et le cosmique [...].»

Jean-Pierre Dubost: «Ubu automobile». Revue des sciences humaines. Lille. no 203. juillet-septembre 1986, p. 167.

EX. - L'article ici cité. 


\section{Textualisation, textualiser}

RÉF. - Raymonde Debray-Genette, 1977; Jean Ricardou, 1982

DÉF. — « «'écrivain n'est constitué que par le fait qu il écrit et quil se lit lui-même. Dès lors qu'un autre le lit, ou qu'il se lit pour un autre (et, bien sûr, sa lecture est toujours et déjà informée par celle des autres), il cherche à constituer son écriture en texte. C'est pourquoi, d'un point de vue génétique, et contrairement à ce que dit R. Barthes, il semble utile de distinguer les phénomènes d'écriture des phénomènes de textualisation, et de considérer le texte comme le produit historique de l'écriture, organisée en commencement et fin, voire finalité."

Raymonde Debray-Genette: "Génétique et poétique: esquisse de méthode». Littérature. Paris. no 28 (no intitulé Genèse du lexte), décembre 1977. p. 29.

«[... la divergence qui distingue sans retour l'expression et la textualisation. Pour la première, toute formule tend à mettre directement en rapport avec un vouloir-dire. Bref, elle fomente un non-lieu idéal. Pour la seconde, toute formule tend à se mettre en contact avec au moins une autre du même écrit. Bref, elle aimante un fort lien matériel.» Jean Ricardou: Le théatre des métamorphoses. Coll. "Fiction \& Cie», Paris. Seuil. 1982. p. 76.

\section{Métatextualisation}

RÉF. — Jean Ricardou, 1982

DÉF. — «[...] une métatextualisation dans le texte. En effet, il sagit bien d'un fragment qui étudie tel mécanisme du texte dans lequel il se situe. Ce fonctionnement, c'est l'abordage frontal par lequel un propos théorique se trouve assailli, à limproviste, par une phrase de fiction.»

Jean Ricardou: «L'escalade de l'autoreprésentation». Texte. Toronto, no 1 (no intitulé Lautoreprésentation. Le texte et ses miroirs). 1982. p. 21.

EX. - Mème article, où il analyse (p. 20-23) des passages de son * milletexte (ou mixte), Le théâtre des métamorphoses.

>> Toujours dans le même article, et comme le dit bien son titre (avec le mot "escalade»), il y a plusieurs degrés et échelons qui mènent, rien de moins, à l' "homométatextualisation" et à l' "hétérométatextualisation» (p. 22-23)! Voir, toutefois, ${ }^{*}$ homotextualisation et * hétérotextualisation. 


\section{Homotextualisation}

RÉF. - Jean Ricardou, 1982

DÉF. - «[...] une homotextualisation du* métatextuel. Ou, si l'on aime mieux, la soumission du * métatextuel à la règle même quili théorise."

Jean Ricardou: "L'escalade de l'autoreprésentation». Texte. Toronto, no 1 (no intitulé Lautoreprésentation. Le texte et ses miroirs), 1982, p. 21-22.

EX. - Voir * métatextualisation.

\section{Hétérotextualisation}

RÉF. - Jean Ricardou, 1982

DÉF. - "[...] une hétérotextualisation du * métatextuel. Ou, si l'on préfère, la soumission du métatextuel à une règle du texte autre que celle qu'il théorise."

Jean Ricardou: "L'escalade de l'autoreprésentation". Texte. Toronto, no 1 (no intitulé Lautoreprésentation. Le texte et ses miroirs), 1982, p. 21.

EX. - Voir * métatextualisation.

\section{Syntextualisation}

RÉF. - Jean Ricardou, 1978

DÉF. - «ll est clair en effet, par opposition au «livre» de Flaubert et au roman [intitulé Martereaul de Sarraute, que Salut. Un coup de dés et la note de Quant au liure [trois écrits de Mallarmél sont respectivement associés: ils appartiennent à un autre système * intertextuel, le * graphotexte, ou ensemble des textes assumés par un même signataire. Ce qui s'est accompli, en conséquence, très précisément, c est la syntextualisation d'un * graphotexte, c'est-à-dire la construction d'un nombre remarquable de relations entre un texte et une autre espèce $d^{\prime *}$ intertexte restreint. (...) Avec la contruction syntextuelle, $l^{* *}$ intertexte général joue un rôle passif: celui de réservoir. Avec la production syntextuelle, $I^{* *}$ intertexte restreint accomplit une fonction active: celle en quelque façon, du texte lui-mème."

Jean Ricardou: Nouveaux problèmes du roman. Coll. «Poétique». Paris. Seuil. 1978. p. 306-307. 
EX. Le passage ici cité.

\section{Cotextualisation}

RÉF. - Leo H. Hoek, 1981

DÉF. - «Le double mouvement entre le titre et le * co-texte détermine entièrement les sens que peut prendre le titre par suite de la lecture. [...] Le ${ }^{*}$ co-texte fonctionne comme une composante transformationnelle qui change le sens fourni par l'input, le titre. À la fin du processus transformationnel que constitue la lecture du ${ }^{*}$ co-texte, elle produit l'output, le titre représentant la structure profonde du * co-texte. Le titre est donc sujet à une modification provenant de la confrontation avec le ${ }^{*}$ co-texte qui transforme non seulement le titre mais aussi le ${ }^{*}$ co-texte $[. .$.$] . Le double mouvement produit un$ double effet: transformation du titre et transformation du co-texte. $\lceil\ldots\}$

Nous distinguons plusieurs types [cinq, en fait] de transformations. La transformation niest jamais nulle car il y a toujours cotextualisation des sèmes que contient le titre, cadrant les sens quili peut prendre; c'est le degré zéro (le premier des cinq types, donc) de la transformation."

Leo H. Hoek: La marque du titre. Dispositifs sémiotiques dine pratique textuelle. La Haye. Paris. New York. Mouton. 1981. p. 180.

EX. — Le livre ici cité.

\section{Métatextème}

RÉF. - Heinrich F. Plett, 1981

DÉF. — «Les figures * textologiques (ou «métatextèmes») se distinguent des autres par le fait qu'elles quittent le cadre étroit de la linguistique moderne. Ceci est déjà évident dans le cas des licences. La figure d'addition * textologique s'appelle "digression" (digressio) et consiste à se détourner du thème principal et à introduire une thématique supplémentaire qui est plus ou moins liée au thème principal (par exemple: A Tale of a Tub de Swift). Dans Tristram Shandy de Sterne un chapitre vide concrétise par exemple la figure de soustraction textuelle: l'auteur respecte certes l'ordre des chapitres, mais laisse vierges des pages de certains chapitres (par 
exemple les chapitres IX. xviii et xix). Comme figures de substitution, nous avons déjà cité l'allégorie (forme textuelle: la parabole) et la périphrase (forme textuelle: la devinette). Enfin la permutation textuelle est parfaitement illustrée par la rupture de l'ordre logique ou chronologique, comme l'exige le principe de l'ordre artificiel (ex. Tristram Shandy). Tous ces exemples mettent en évidence le fait que les écarts * textologiques relèvent plutôt de l'étude théorique des structures narratives (en tant que "figures narratives») que de la linguistique. Mais l'exemple de l'équivalence montre que le genre lyrique et le genre dramatique sont tout aussi bien empreints de figures * textologiques. Toutes les fois que paraissent en poésie des refrains qui dépassent le cadre phrastique, il s'agit de figures de répétition ${ }^{*}$ textologiques. On trouve un cas extrême d'équivalence dramatique dans Play de Beckett, où le texte entier est répété. Il ressort de tout cela que les figures ${ }^{*}$ textologiques déterminent la structure (dispositio) des textes et qu'ainsi un trait d'union subsiste entre la théorie du style et la théorie du genre.

Heinrich F. Plett: «Rhétorique et stylistique" dans Aron Kibédi Varga (sous la direction de): Théorie de la littérature. Coll. «Connaissance des langues», Paris, Picard, 1981, p. 171.

>> > On remarquera que H.F. Plett écrit indifféremment * textologique (comme dans «addition textologique») et textuelle (comme dans "permutation textuelle»), utilisant le premier terme au sens où $\mathrm{J}$. Seebacher aimerait qu'il soit utilisé: voir, sur ce point précis, ${ }^{*}$ textologie et la note de R. Laufer.

>> L'article ici cité présente un «bref aperçu des classes des figures (sémio-)syntaxiques" (p. 171) selon les six plans linguistiques suivants: figures phonologiques (ou «métaphones»), morphologiques (ou "métamorphes»), syntaxiques (ou "métataxes"), sémantiques (ou "métasémèmes»), graphématiques (ou «métagraphes») et textologiques (ou «métatextèmes»).

\section{Antitexte}

RÉF. — Leo H. Hoek, 1981; Jan Baetens, 1986-1987

DÉF. - Leo H. Hoek, parle d'antitexte sans vraiment développer davantage que ceci: «Les anti-textes portent depuis toujours des titres comme Anti-Huguenot (Reboul), (...) Lanti-roman (Sorel), (.... Antimémoires (Malraux), Anti-manuel de français (Duneton et $\mathrm{Pa}$ gliano)."

Leo H. Hoek: La marque du titre. Dispositifs sémiotiques diune pratique terluclle. La Haye. Paris. New York. Mouton, 1981, p. 6. 
$\S$ Dans «Zigzag» (chapitre de Jean-Claude Lebensztejn: Zigzag, coll. "La philosophie en effet", Paris, Flammarion, 1981 l, la mise en page n'est pas donnée une fois pour toutes. Le schéma cité ci-dessus se modifie au fur et à mesure que progresse le livre [...] et cela justement sous l'influence et la pression de ce qui l'excède, de ce qui déroge à ses principes. Car les exceptions, chez Lebensztejn, ne se contentent pas de contredire la règle: elles ne sont admises que pour amener une transformation de cette règle, et c'est là, finalement, toute la différence entre La crue [de Lucette Finas, coll. «Le chemin», Paris, Gallimard, 1972l et Zigzag: là, l'exception reste étrangère au dispositif: ici, le dispositif provoque des ruptures pour atteindre à un degré d'organisation et de complication supérieur.

Cette démécanisation [est] opérée par la * textualisation de la faute (par la * textualisation de l'antitexte, si l'on veut) (...).» Jan Baetens: "Autres figures du discours". Texte. Toronto. no 5/6 (no intitulé Théories du (exte), 1986-1987, p. 246.

>> L'antitexte selon Baetens est une région de la * grammatextualité.

EX. - L'article ici cité.

\section{Sociotexte}

RÉF. - Jean-Jacques Thomas, 1977 - «Pragmatique et sociotexte», dans Claude Duchet (sous la direction de): Sociocritique (actes du colloque de Vincennes, novembre 1977), Paris, Nathan, coll. "Nathan-Université», 1979, p. 45-50.

> > Ce bref article ne contient pas, à proprement parler, une définition du terme en question. La définition que nous choisissons est celle que propose Pierre V. Zima (qui, par ailleurs et à notre connaissance, n'emploie pas ce terme) de la sociologie du texte.

DÉF. - «À la différence des méthodes existantes en sociologie de la littérature qui s'orientent vers des aspects thématiques ou «idéels" de "loeuvre", la sociologie du texte s intéresse à la question de savoir comment des problèmes sociaux et des intérêts de groupe sont articulés sur les plans sémantique, syntaxique et narratif."

Pierre V. Zima: Manuel de sociocritique, Coll. "Connaissance des langues», Paris, Picard, 1985, p. 9.

>> On pourrait donc envisager une définition de ce terme qui serait à la sociologie ce que l'inconscient du/dans le texte est à la psychana- 
lyse: voir * textanalyse selon J. Bellemin-Noël. Bien que Pierre V. Zima nutilise pas ce terme, il nous semble cependant que ce qui est articulé (problèmes sociaux et intérêts de groupe) par le texte (plans sémantique, syntaxique et narratif) pourrait bien s'appeler un sociotexte.

EX. - Ce livre de Pierre V. Zima, particulièrement le chapitre 4 de la seconde partie (p. 115-185).

\section{Contexte}

RÉF. - Louis Bloomfield, 1933 - Le langage, Paris, Payot, 1970 [1 ère édition américaine: 1933]

DÉF. - «On appelle contexte l’ensemble du texte qui précède et/ou qui accompagne l'unité syntagmatique considérée, et dont dépend la signification. Le contexte peut être explicite ou linguistique, ou bien implicite et qualifié, en ce cas, dextralinguistique ou de situationnel. Le contexte implicite peut être exploité en vue de l'interprétation sémantique [...].»

Algirdas J. Greimas et Joseph Courtés: Sémiotique. Dictionnaire raisonné de la théorie du langage. Coll. "Langue linguistique communication». Paris, Hachette, 1979. p. 66-67.

>> U Une fois n'est pas coutume: voilà que nous venons de citer un dictionnaire! Cette définition du terme "contexte» pourrait, de fait et sur le modèle genettien de * péritexte/* épitexte, engendrer "péricontexte» (ou contexte textuel: microcontexte ou contexte restreint, macrocontexte ou contexte élargi) et "épicontexte" (ou contexte * extratextuel: sociohistorique, sociopolitique, biographique, etc.).

\section{Contexture}

RÉF. - Nicolas Abraham, 1948 (publié en 1985)

DÉF. - «ll faut entendre par là l'ensemble des circonstances situation, atmosphère, ${ }^{*}$ contexte, etc. - qui entre en synthèse avec le medium pour en déterminer la signification. Le malentendu, c'està-dire la différence des objets intentionnés par deux sujets à travers des contenus significatifs identiques, tient le plus souvent à une différence des contextures dans lesquelles, des deux parts, vient 
s'incorporer le même medium. L'objet que donne le medium est donc fonction non seulement de la nature de celui-ci, mais également de la contexture. Si tout contenu, quel qu'il soit, peut servir de medium (puisquil n'est pas de contenu qui ne puisse renvoyer de quelques manières à d'autres contenus), le medium, en tant que medium de tel objet, demeurera plus ou moins indéterminé et conservera une certaine polyvalence significative, tant qu'une synthèse appropriée ne l'aura intégré dans une contexture déterminante."

Nicholas Abraham: Rythmes. De l'oeuvre de la traduction el de la psychanalyse. édition de Nicholas T. Rand et Maria Torok, coll. "La philosophie en effet". Paris, Flammarion, 1985, p. 17-18.

\section{Grammatextualité}

RÉF. - Jean Gérard Lapacherie, 1984

DÉF. - «La grammatextualité est la propriété qua l'écriture (indépendamment peut-être du discours oral) de constituer des textes, selon des "lois" sémiotiques qui lui sont propres et que l'on va s'efforcer de déterminer plus bas.

Est posée dans les lignes qui suivent l'hypothèse d'une «réactivation" des signifiants graphiques, pour parler comme R. Jakobson, de sorte que l'écriture - lettres, lignes, blancs, dispositifs de mise en pages, tout ce qui apparait sur la page, ainsi que le support du texte cesse d'être la simple manifestation visuelle (mais que l'on ne voit plus) d'un discours premier. À la "fonction poétique» du langage $[. .$. pourrait correspondre une fonction grammatique (ou fonction poétique de l'écriture), définie provisoirement et en termes jakobsoniens [...] comme l'accent mis sur le graphisme scripturaire pour lui-même, indépendamment de la substance phonique et de l'enchaînement discursif.

(...) La grammatextualité, propriété commune à toute une série de textes, «littéraires" ou non, "poétiques» ou non, recouvre plus des pratiques d'écriture comme la figuration par la lettre, les dispositifs fragmentaires ou foisonnants, les figures de rhétorique graphique, les faits de "mimographisme»..., que des textes achevés. Elle englobe aussi les pratiques scripturaires qui utilisent "toutes les ressources de la typographie", qui est à la fois un art de la lettre et de la mise en pages et une écriture seconde dérivée.

(...)

Quatre degrés de figuration scripturaire, auxquels il est possible provisoirement de se limiter, ont été isolés: l'icône, l'idéogramme, le diagramme et l'alphabet, quatre principes différents de représenta- 
tion par l'écriture. Ces principes, à l'oeuvre dans les pratiques grammatextuelles, constituent une des potentialités de l'écriture.

[...] Trois des quatre degrés de figuration, le diagramme, l'idéogramme, l'alphabétique, recouvrent des pratiques langagières et scripturaires. Le quatrième, l'icône, est uniquement scripturaire. Autrement dit, cette opposition langage us non-langage en détermine une autre, plus fondamentale, entre le langage et l'image. L'iconique, essentiellement figuratif, mais d'une figurativité scripturaire, relève d'une sémiologie de l'image.»

Jean Gérard Lapacherie: «De la grammatextualité». Poétique. Paris, no 59, septembre 1984, p. 283-284, 288 et 291.

EX. - Jean Gérard Lapacherie («Écriture et lecture du calligramme», Poétique, Paris, no 50, avril 1982, p. 194-206) analyse des calligrammes d'Apollinaire.

\section{Callitexte}

\section{RÉF. — Daniel et Nicole Bilous, 1986}

DÉF. - «On a vu que d'une part, certains mécanismes textuels existaient à l'état sporadique dans le calligramme, et en favorisant la lisibilité; que d'autre part, sous peine de non-représentation, il ne fallait guère envisager de "textualiser»-l'image. C'est par conséquent du côté de l'écrit que devront se porter les efforts de surdétermination productive. Le callitexte, si le concept a quelque chance de trouver un jour son référent, définit une situation où le calligramme «rencontre son autre», c'est-à-dire ce que précisément il a censuré pour advenir: l'axe monolinéaire orienté de l'écrit.»

Daniel et Nicole Bilous: «Lire le calligramme». Protée. Chicoutimi, vol. 14, no 1-2 (no intitulé La lisibilité), printemps-été 1986, p. 125.

EX. - L'article ici cité, où sont analysés des calligrammes de G. Apollinaire, M. Leiris et des auteurs.

\section{Textanalyse}

RÉF. - Jean Bellemin-Noël, 1979

DÉF. - «Ayant mis l'homme, l'auteur, entre parenthèses, visant à élaborer en critique la méthode d'une lecture psychanalytique des 
textes - d'une textanalyse - , on a constaté que je posais les hypothèses suivantes: tout texte est travaillé par un discours inconscient; il est possible de décrire ce travail qui s'effectue dans le texte; cette description n'a pas pour objet une traduction, mais la reconnaissance d'un fonctionnement oblique du texte comme force engagée dans l'oeuvre d'écriture; il ne suffit pas pour conduire cette analyse de recourir aux concepts de Freud et de ses disciples, il faut faire retour sur eux, car la lecture littéraire déplace une théorie axée sur la clinique ou sur la compréhension générale du phénomène humain. Voilà dans quel contexte de questions et de positions j'ai commencé, en 1970, à parler d'inconscient du texte len note, J. Bellemin-Noël ajoute: L'expression m'a été imposée, non par hasard sans doute, au cours d'une étude de genèse utilisant les brouillons d'un poème; v. Le texte et lavant-texte»l, voilà pourquoi je continue d'employer cette expression, à laquelle recourent également d'autres critiques.»

Jean Bellemin-Noël: Vers linconscient du texte. Coll. «Écriture», Paris, PUF, 1979. p. 191.

«Parlons clair: selon mes propres concepts, quelque chose comme une «avant-textanalyse» n'est simplement pas pensable en toute rigueur. Dans la pratique, ou bien l'on adjoint l* avant-texte au texte, c'est-à-dire que l'on ajoute aux diverses structures de signification des données provenant de l'histoire de ce signifié, donc que l'on complète des éléments coextensifs par des éléments antérieurs, - et alors on a tout uniment affaire à du texte, $l^{*}$ avant-texte perdant sa spécificité (cf. le cas des variantes au sens strict). Ou bien l'on se préoccupe de genèse, - et alors il faut outrepasser le textuel en direction du procès d'écriture et remonter jusqu'à l'écrivain. Cette logique est valable pour les analyses du texte qui ont d'autres visées que psychanalytiques et qui, tout autant que nous, ont à gagner aux recherches génétiques.»

Jean Bellemin-Noël: «En guise de postface: l'essayage infini».Littérature. Paris, no 52 (no intitulé L inconscient dans lavant-texte), décembre 1983, p. 126.

EX. - Ce numéro de Littérature analyse des avant-textes de V. Hugo, J. Giono, A. de Vigny et G. Flaubert.

\section{Textologie, textologique}

RÉF. - Boris V. Tomachevski, 1928 - L'écrivain et le livre. Esquisse de textologie, Léningrad, 1928 


\section{DÉF. - «Textologie: science du texte}

La textologie étudie les conditions générales d'existence des textes. Les paroles s'envolent, les écrits demeurent: mais ni les textes, ni les lectures n'échappent aux atteintes du temps. La lettre s'altère, l'esprit change. La textologie s'efforce d'assurer la bonne transmission de certains messages. Elle fait donc partie des sciences de la communication, comme la philologie classique et médiévale, dont elle se distingue par une extension moindre (portant sur des textes mieux préservés et culturellement plus proches), un développement modeste (la philologie classique accumule le savoir de nombreux siècles), mais une ouverture majeure à un problème d'aujourd'hui: la transformation de la culture liée aux moyens de communication de masse, la mise en question de la typographie et de l'écriture.

\section{Situation de la textologie}

Le mot s'emploie couramment dans les pays de langues slaves. Il a été créé dans les années vingt par Tomachevski et employé par lui dans le sous-titre d'un livre publié à Léningrad en 1928, Lécrivain et le liure. Esquisse de textologie IR. Laufer ajoute en note: Ce mot existe dans une acception précise depuis un demi-siècle. Jacques Seebacher l'a réinventé et employé dans le sens d'analyse textuelle. Sans débattre du fond et pour la seule clarté terminologique, il semble juste, si on adopte le mot, de se conformer à l'usage scientifique international. / Ces précisions ne sont pas indifférentes. Elles appellent un double commentaire.

La textologie s'est constituée comme discipline autonome dans la première décennie du pouvoir soviétique, c'est-à-dire à une époque où les acquis de l'érudition ont été appliqués à la diffusion de masse: l'art d'éditer les textes servait une révolution culturelle. Si le terme n'a pas été adopté dans les pays occidentaux, c'est que les conditions économiques ne permettaient pas aux chercheurs et aux grandes maisons d'édition de collaborer. L'éditeur de textes y est de nos jours encore moins bien rémunéré que le traducteur, ce qui en dit assez; et le fruit de son labeur n'est pas protégé contre les contrefaçons.

L'édition des textes modernes a cependant été renouvelée dans les pays anglo-saxons par le développement de la bibliographie matérielle, qui semble, sinon peu connue, du moins peu pratiquée en Union Soviétique. L'importance des études shakespeariennes explique en partie ces progrès, mais aussi l'institution des congés de recherche et l'existence de Presses d'Universités.

$\lceil\ldots\}$

Le texte fonde toute étude littéraire. Cette constatation n`abroge pas la nécessaire référence de toute critique littéraire au dehors du texte ( ${ }^{*}$ contexte, ${ }^{*}$ hors-texte, ${ }^{*}$ méta-texte) sur le fond duquel celuici se situe pour accéder à la signification. (...) 
La textologie se situe donc dans l'étroit domaine qui sépare les textes d'eux-mêmes et ne départage qu'une frange de leur signification. Ceci ne doit pas surprendre: rétablir un texte est une activité tautologique, le moderniser consiste seulement à en modifier certaines normes à l'intérieur du code graphique. La textologie échoue dans les cas difficiles: transmission orale, textes inachevés, brouillons, etc., où on ne veut accomplir la tâche séduisante mais strictement irréalisable de * textualiser du non-texte. (...)

La textologie veille à la bonne utilisation des signes typographiques: elle ne s'applique qu'à des textes déjà typographiques ou du moins pré-typographiques, c'est-à-dire compatibles avec le système. Elle est une sémiologie scientifique des textes parce qu'elle néglige la signification humaine, philosophique, etc. au profit du sens opératoire des signes en tant qu'ils fondent l'espace de la * textualité.»

Roger Laufer: Introduction à la textologie. Vérification. établissement. édition des textes. Coll. «L», Paris, Larousse, 1972, p. 5-6 et 8-9.

EX. - Toute édition dite critique.

>> Dans ce livre, ce terme, et tous les autres auxquels il est joint, ont un sens technique précis. Ainsi «avant-texte», "sous-texte» et "aprèstexte» (p. 74-75) désignent simplement la localisation de l'appareil éditorial: (préface) avant le texte édité, (notes) au bas des pages du texte édité, (notes rejetées) après le texte édité. Par ailleurs, "architexte» (p. 75) désigne l'architecture générale (disons La comédie humaine) dans laquelle s'inscrit une oeuvre (disons Le cousin Pons) et «non-texte», on le voit, est défini dans le passage ici cité. Voir aussi "texte de base" ( ${ }^{*}$ texte souche). C'est dire quils ne font pas sens pour la théorie du texte. Le textologue, ici, est l'antagoniste du * textualiste. Enfin, R. Laufer discute (p. 84) de la position, elle aussi antagoniste, de J. Bellemin-Noël (sur le terme * avant-texte particulièrement).

\section{Texte source (Texte souche, Texte matrice)}

RÉF. - ?

DÉF. - Voir * hypotexte.

>> Texte de base, en * textologie, désigne cette «réalisation concrète (manuscrite ou imprimée) qui servira de fondement à l'établissement [du texte]» (R. Laufer: Introduction à la textologie, p. 17 - voir aussi, p. 17-35 et 45-52). 
Texte cible [Texte objet]

RÉF. - ?

DÉF. - Tout texte qui est l 'objet d' un regard théorique et/ou critique. 